\title{
Diseño teórico de terapia combinada con un inhibidor de BMPR y un miRNA contra RUNX-2 en la Enfermedad de la Válvula Aórtica Calcificada.
}

\author{
Elisa San Isidro ${ }^{a}$, Elena de la Torre-Rubio ${ }^{b}$, Judith Recioc, Alberto Cook ${ }^{d}$ \\ Unidad de Bioquímica y Biología Molecular, Departamento de Biología de Sistemas, Facultad de Medicina y Ciencias \\ de la Salud, Universidad de Alcalá, 28871 Alcalá de Henares, Madrid, España.
}

a. elisasanisidro@gmail.com b. delatorrerubio.elena@gmail.com c. judithrecio1997@gmail.com

d. mcooky_8@hotmail.com

V Congreso de Señalización Celular, SECUAH 2020

Departamento de Biología de Sistemas, Facultad de Medicina, Universidad de Alcalá (UAH). Alcalá de Henares, Madrid (Spain)

Palabras clave: EVAC; Liposoma; BMP2; RUNX-2; miRNA; péptido-mimético

\section{Resumen}

La enfermedad de la válvula aórtica calcificada (EVAC) es una enfermedad caracterizada por la diferenciación de las células intersticiales de la válvula hacia un fenotipo pre-osteoblástico. Esta diferenciación celular produce una matriz calcificada, causando de esta forma un remodelaje tisular que conlleva a la estenosis aórtica y a una pérdida de la funcionalidad. Nuestro proyecto se enfoca en evitar la diferenciación osteoblástica inhibiendo la cascada de señalización de BMP2 y el factor de transcripción RUNX-2. Para ello planteamos el siguiente diseño teórico de la investigación que se realizaría para llevar a cabo el descubrimiento de 2 posibles fármacos empleados sinérgicamente en una terapia combinada.

Cita: San Isidro, Elisa; de la Torre-Rubio, Elena; Recio, Judith; Cook, Alberto (2020) Diseño teórico de terapia combinada con un inhibidor de BMPR y un miRNA contra RUNX-2 en la Enfermedad de la Válvula Aórtica Calcificada. Actas del V Congreso de Señalización Celular, SECUAH 2020. Departamento de Biología de Sistemas, Facultad de Medicina, Universidad de Alcalá (UAH). Alcalá de Henares, Madrid (Spain). dianas 9 (1): e202003fa02. ISSN 1886-8746 (electronic) journal.dianas.e202003fa02 http://www3.uah.es/dianas?e202003fa02. URI http://hdl.handle.net/10017/15181

Copyright: ( $)$ San-Isidro E, de-la-Torre-Rubio E, Recio J, Cook A. Algunos derechos reservados. Este es un artículo open-access distribuido bajo los términos de una licencia de Creative Commons ReconocimientoNoComercial-SinObraDerivada 4.0 Internacional. http://creativecommons.org/licenses/by-nc-nd/4.0/

\section{Introducción}

Las enfermedades cardiovasculares (ECV) son un conjunto de patologías caracterizadas por un desorden del corazón y de los vasos sanguíneos. Dentro de las ECV, la enfermedad de la válvula aórtica calcificada (EVAC) es la valvulopatía con mayor índice de morbimortalidad en los países desarrollados [1]. La EVAC es un desorden crónico caracterizada por una fibrosis y mineralización ectópica de la válvula aórtica (VA), la cual suele desembocar en estenosis aórtica (EA) [2].

Actualmente no existen estrategias terapéuticas no invasivas para prevenir o tratar la EVAC. Aunque el advenimiento de las nuevas tecnologías de reemplazo valvular ha mejorado los resultados clínicos, estas técnicas siguen siendo subóptimas para las dos poblaciones con mayor riesgo de complicaciones valvulares: pacientes pediátricos y ancianos [3]. Por ello, es necesario ahondar en los mecanismos patológicos que subyacen durante la enfermedad y poder así identificar los biomarcadores tempranos de la enfermedad para aplicar nuestro tratamiento a tiempo de forma eficaz. Además, entender cómo se desarrolla la patología a nivel celular nos ayudará a identificar algún marcador específico del tejido afectado para dirigir nuestro tratamiento de forma selectiva.

\section{Mecanismos patológicos}

Se han descrito dos hipótesis de morfogénesis del nódulo calcificado de la población de células intersticiales de la válvula (VICs) residentes: la teoría de la calcificación apoptótica o distrófica y la teoría de la osificación. El evento principal de la teoría de la calcificación distrófica es la calcificación de células muertas o dañadas que se conoce y se considera como un fenómeno pasivo. Sin embargo, la calcificación se acelera por la edad y el aumento del estrés mecánico. Se ha observado que el factor de crecimiento transformante de citoquinas $\beta 1$ (TGF- $\beta 1$ ) induce una calcificación distrófica rápida in vitro a través de un proceso mediado por apoptosis [4-7]. Por el contrario, las proteínas morfogenéticas óseas 2 y 4 (BMP-2 y BMP-4) son potentes morfógenos osteogénicos que están asociados con la teoría de la osificación, que especifica la diferenciación osteogénica regulada de las VICs en lugar de la lesión celular como el evento principal, y por lo tanto una calcificación activa [8-12]. No está claro si estos procesos de calcificación pueden ocurrir independientemente uno del otro, pero sí pueden ocurrir simultáneamente ya 
que se ha encontraron que el núcleo de los nódulos contiene diferentes tipos de mineralización, así como células apoptóticas [13]. Además, se describieron nódulos osificantes que no contenían células apoptóticas, señalando que se pueden estar formando diferentes tipos de nódulos [14].

Otros estudios han asociado la activación de las VICs con un aumento de la secreción y degradación de la matriz extracelular (ECM), la expresión de metaloproteinasas (MMP) e inhibidores tisulares de MMP (TIMP) importante para la reparación de heridas [15]. Además, se ha observado que la remodelación de la ECM conduce a una mayor liberación de TGF- $\beta$ [16]. Curiosamente, la aplicación combinada en los primeros estadíos de la enfermedad de TGF- $\beta$ y estrés cíclico a las valvas de la válvula aórtica en condiciones osteogénicas ex vivo conduce a un aumento expresión de BMP-2 y BMP-4 y de citoquinas asociadas con la osteogénesis progresiva. Estas observaciones sugieren que tanto las vías miofibroblásticas como las osteogénicas pueden contribuir de forma secuencial a la EVAC.

Nuestro estudio consiste en diseñar una terapia combinada para prevenir la diferenciación celular de las VICs y poder así evitar la calcificación de la válvula. Para ello es imprescindible identificar los cambios tempranos que se producen en las células de la válvula en los primeros estadios de la enfermedad, para poder aplicar un tratamiento menos invasivo y regenerativo, y si fuera posible, que pudiera revertir el proceso patológico. Estudios demuestran [3] que la remodelación que se produce en la matriz extracelular podría ser una ventana terapéutica para poder diagnosticar precozmente la enfermedad.

\section{Liposomas}

Después de que un medicamento ingresa a la circulación sistémica, no se distribuye uniformemente a los tejidos del cuerpo debido a las diferencias en la perfusión de la sangre del órgano, la afinidad del tejido, el $\mathrm{pH}$ local y la permeabilidad de la membrana celular.

Una de las vías de transporte de medicamentos propuesta en numerosos estudios es mediante el uso de liposomas. Algunas de las ventajas principales de usar los medicamentos atrapados en liposomas es la especificidad por su diana terapéutica y la reducción de la toxicidad de los propios medicamentos ya que éstos no están biodisponibles hasta que se liberan al órgano diana. Además, los liposomas se pueden diseñar de tal forma que puedan ser captados de forma selectiva atendiendo a los receptores específicos de membrana de la célula diana. De esta forma se mejoran los resultados terapéuticos al aumentar la eficacia y reducir la toxicidad fuera del objetivo [17].

Numerosos estudios presentan la versatilidad de los liposomas en cuanto a sus vías de administración, ya que además de su posible uso sistémico, es decir, la administración intravenosa [18], los liposomas se han diseñado para la administración en aerosol [19] o intradérmica [20]. Estudios actuales llegan a presentar la posibilidad incluso de una administración oral mediante la manipulación de la envuelta lipídica de los liposomas $[21,22]$.

\section{Biomarcadores diagnósticos}

Mientras que la ecocardiografía es una técnica incapaz de poder identificar los estadios más tempranos de la enfermedad, recientemente las técnicas de imagen por PET/CT han surgido para poder detectar estados de inflamación y mineralización en los tejidos cardiovasculares. Además, la imagen molecular óptica ha resultado ser un éxito para poder visualizar la progresión de la EVAC en ratones [23-25]. Utilizando esta tecnología de imagen podríamos observar mejor la progresión temporal de la EVAC y liderar de una manera más específica los requerimientos terapéuticos de cada estadio de la enfermedad.

Estos tratamientos más dirigidos contra la diferenciación patológica de las células de la válvula aórtica y la remodelación de la matriz extracelular podrían suponer un importante y desconocido jardín terapéutico.

Dentro de los diferentes estadios de la EVAC, los procesos patológicos que acompañan y caracterizan la patocronia de la enfermedad son: el metabolismo de lípidos y estrés cardíaco, el metabolismo mineral y de la formación del hueso y la inflamación.

\section{Metabolismo de lípidos y estrés cardíaco}

Las lipoproteínas plasmáticas que contienen apo-B podrían jugar un rol importante en la patogénesis de la EVAC [26].

El estrés mecánico del flujo sanguíneo a través de la válvula aórtica del corazón podría ser capaz de dañar la válvula, aumentando la susceptibilidad de penetración de dichas lipoproteínas al endotelio vascular. Estas partículas son responsables de la modificación oxidativa del intersticio de la válvula ya que contienen un alto número de lípidos oxidados en su interior. Además, se ha observado histológicamente que los tejidos afectados presentan infiltrados de células inflamatorias; lipoproteínas y lípidos que se relacionan con la estenosis de la válvula aórtica del tejido. Mientras que algunos estudios correlacionan una asociación entre el colesterol total y la cantidad de triglicéridos con la EVAC durante los estadios más tempranos de la enfermedad [27,28] los niveles de HDL no parecen demostrar diferencias 
significativas. Sin embargo, la lipoproteína A (la cual contienen apo-B) sí parece guardar una relación estrecha con la patogénesis de la calcificación durante la enfermedad.

\section{Metabolismo mineral y óseo}

La deposición de Calcio y mineralización ósea son cambios anatomopatológicos que se relacionan con los últimos estadios de la enfermedad, comprometiendo el correcto funcionamiento de la válvula. El mecanismo de formación del hueso se debe a un cambio en el fenotipo de las VICs hacia una diferenciación osteoblástica, los cuales coordinan la calcificación de una forma similar a la que se produce en el hueso.

Los polimorfismos del receptor de la vitamina D y los niveles altos de la concentración de fosfato en plasma con enfermedad renal se han visto asociadas al desarrollo de la calcificación valvular [29].

Se distinguen biomarcadores en 2 subgrupos: biomarcadores específicos de metabolismo mineral y biomarcadores específicos para la formación y resorción del hueso.

\section{i. Biomarcadores del eje Vitamin D-Hormona paratiroidea:}

Los biomarcadores del metabolismo mineral se refieren principalmente a la vitamina D y la hormona paratiroidea $(\mathrm{PTH})$. Se incluye por lo tanto el calcio y fosfato en plasma, vitamina $\mathrm{D}$, factor de crecimiento de fibroblastos (FGF-23), y la PTH. El eje vitamina D-hormona paratiroidea se ha visto que está implicada en la EVAC. Los niveles de calcio iónico y fosfato en plasma están finamente controlados por la activación de la vía vitamina $\mathrm{D}$ por el riñón y la secreción de la PTH por las glándulas paratiroideas, y la generación de FGF-23 por los osteocitos. Se sospecha que la desregulación en los niveles de calcio y fosfato en plasma puedan promover el desarrollo de la EVAC. Hay estudios que han observado que mientras que los niveles de calcio, PTH y FGF-23 en plasma no se puedan correlacionar con un aumento en la calcificación, los niveles de fosfato en plasma sí se ven alterados [30,31].

ii. Biomarcadores de la formación y resorción del hueso:

El segundo grupo de biomarcadores del metabolismo mineral del hueso son aquellos que se tienen una expresión diferente anómala durante alguna patología generando una formación y resorción del hueso anormal. Entre estos biomarcadores nos encontramos el propéptido N-terminal de procolágeno tipo I humano (PINP), osteocalcina, osteopontina, osteoprotegerina y fetuin-A entre otros. La osteopontina, osteoprotegerina y fetuin-A son marcadores inflamatorios que afectan a la deposición de calcio. La osteopontina es una glicoporteína proinflamatoria que inhibe la deposición de calcio por los osteoblastos. La osteoprotegerina se sabe que es un factor de riesgo para la aterosclerosis progresiva y enfermedades cardiovasculares, ya que es capaz de unirse (como cebo, sin producir señalización), a los receptores RANK para inhibir la diferenciación del precursor de osteoclastos [32].

Fetuin-A es una glicoproteína sintetizada por el hígado en circulación por el torrente sanguíneo cuya función es inhibir la calcificación [33]. Hay estudios que demuestran una relación inversa entre los niveles de fetuin-A y calcificación vascular, por ejemplo, en pacientes en estadios finales de enfermedad renal [34]. Por otro lado, los niveles de osteopontina y de osteoprotegerina se muestran incrementados en circulación asociándose con un incremento en la severidad de EA [35]. Además, los niveles de osteoprotegerina aumentan en individuos con fallo cardíacos, resaltando su posible potencialidad como molécula predictora de las consecuencias que ocasiona la necesidad de un remplazamiento valvular. Sin embargo, ni siquiera los niveles de fetuin-A son predictivos para poder diferenciar significativamente la severidad de la EA.

Estos resultados destacan la posibilidad de usar estas moléculas como biomarcadores plasmáticos, asociados con la formación y resorción del hueso, que reflejen la perturbación sistémica durante la EVAC, incluso de la expresión específica valvular de esas proteínas.

\section{Inflamación}

En los últimos años se ha profundizado en la gran evidencia que existen en la asociación entre el rol inflamatorio y la disfunción endotelial en la aterogénesis. Este proceso también se ve involucrado en el tejido valvular aórtico, pudiéndose reconocer un estado inflamatorio durante la EVAC del intersticio valvular similar al que se produce durante la aterosclerosis [36]. Durante la EVAC y la aterosclerosis, la disfunción endotelial es un evento iniciador que promueve la penetración del endotelio tanto vascular como valvular por lípidos oxidados y células inflamatorias [37]. Sin embargo, esta combinación de lípidos oxidados y células inflamatorias en el tejido suponen la generación de moléculas inflamatorias responsables de los procesos de fibrosis y calcificación característicos ya en estadios avanzados de la enfermedad [38].

Uno de los biomarcadores pro-inflamatorios más estudiados en este tipo de patologías es la proteína C reactiva (CRP), la cual se libera durante una fase aguda por el hígado en respuesta a inflamación convirtiéndose en un buen biomarcador de un bajo grado de inflamación. Sin embargo, un elevado CRP 
en suero es un predictor independiente de la EVAC y eventos cardiovasculares, por lo que desafortunadamente varios estudios han reflejado la imposibilidad de correlacionar significativamente los niveles de CRP con la progresión de EVAC. Una de las posibles explicaciones que indican que la CRP no es un buen predictor de dicha enfermedad es que la inflamación no juega un papel muy importante en las primeras fases de la EVAC [39].

El diagnóstico precoz de la enfermedad de la calcificación de la válvula aórtica del corazón es necesario para el desarrollo de una estrategia terapéutica que no implique el reemplazamiento de la válvula. De los biomarcadores mencionados anteriormente se pueden identificar aquellos que se presentan de forma temprana en los inicios de la enfermedad previo a la calcificación. Los biomarcadores que se pueden correlacionar con la EVAC de forma precoz son los niveles de lipoproteína A, los niveles altos de colesterol y fosfato inorgánico u osteopontina. Aquella población de mediana edad que presentara de forma rutinaria estos biomarcadores tempranos de la enfermedad podrían ser susceptibles de desarrollar EVAC en el futuro.

Nuestro diseño de la terapia terapéutica en la EVAC ofrece una alternativa al único tratamiento actual: el reemplazamiento valvular quirúrgico, el cual es un método invasivo y limitado en el tiempo. Para ello profundizaremos en las vías de señalización implicadas en esta patología y explicaremos los distintos ensayos que se deberían realizar para diseñar de forma óptima una molécula selectiva y eficaz contra la diana terapéutica seleccionada.

\section{Vías de señalización implicadas en la EVAC}

La parte interna de la válvula aortica, cuya estructura se muestra en la Figura 1, está compuesta por VICs. En el transcurso de esta patología, en un estadio temprano, dichas células obtienen un fenotipo de miofibroblastos, encargados de remodelar la matriz extracelular. En estadios más avanzados, las células se diferencian a osteoblastos, debido a la expresión de diferentes factores de transcripción, siendo estas células las responsables de la calcificación valvular [2].

\section{Proceso de calcificación}

Al inicio de la enfermedad, y una vez las células VICs se hayan diferenciado a miofibroblastos, se forman agrupaciones de células, llamados nódulos, donde las células que se encuentran más en el interior de estos nódulos mueren por apoptosis. En la matriz extracelular hay presencia de iones calcio que pueden reaccionar con los grupos fosfato de las cabezas de los fosfolípidos que se encuentran en las membranas de estas células apoptóticas, produciéndose la calcificación de estas células muertas [40].

Con el transcurso de la patología, las VICs van a comenzar a diferenciarse a pre-osteoblastos y estas a osteoblastos, esta diferenciación se caracteriza por la expresión de ciertos marcadores moleculares mencionados anteriormente. Los osteoblastos son células capaces de producir la matriz extracelular presente en los huesos, la hidroxiapatita, compuesto formado por fosfato cálcico cristalino que puede producir la calcificación, parecido a lo que ocurre en los huesos [41].

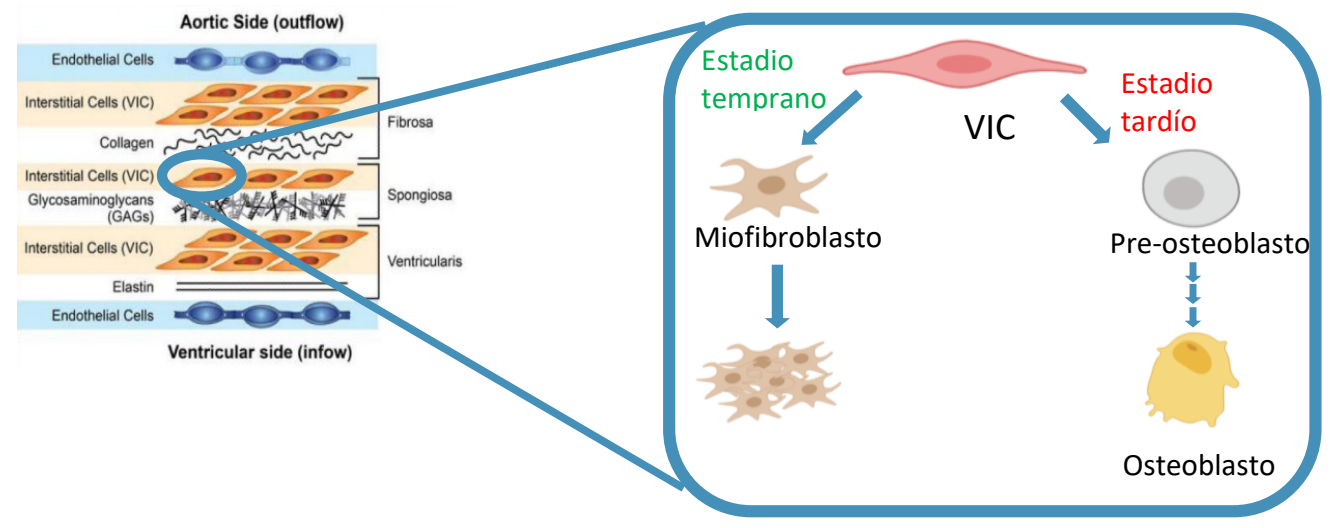

Figura 1.- Estructura a nivel celular de la válvula aortica y diferenciación de las VICs durante desarrollo de la EVAC

\section{Desarrollo de la patología a nivel molecular}

A nivel molecular, como se muestra en la Figura 2, se observa se trata de un proceso bastante complejo debido a la multitud de vías implicadas. Pero la molécula clave de este proceso es RUNX-2, un factor de transcripción asociado con la diferenciación a osteoblastos, y, por tanto, implicado en la calcificación. Por lo que aquellas vías que activan la expresión de RUNX-2, como NFKB, BMP-2 y la vía Wnt, promueven 
la diferenciación a osteoblastos. Y, por el contrario, las vías que inhiben la expresión de RUNX-2, como la vía Notch, son las que evitan esta diferenciación $[42,43]$.

En los estadios iniciales de la enfermedad, se puede apreciar una fibrosis del tejido y se empieza a crear un ambiente pro-inflamatorio en el que, entre otros, se libera TGF- $\beta 1$ que es el principal responsable de la diferenciación de las VICs a miofibroblastos. Este ligando se une a su receptor, estimulando varias vías de señalización. Mediante la vía no canónica, se activa la cascada de las MAPK desencadenando la expresión de cadherina-11. La cadherina-11 es una de las proteínas responsables de la formación de nódulos que acabarán calcificados. En cambio, mediante la vía canónica se activan las proteínas SMAD 2/3, que uniéndose a SMAD 4, son capaces de entrar al núcleo e inhibir la transcripción del gen de RUNX-2, no produciéndose la diferenciación a pre-osteoblastos [44].

En estadios más avanzados de la enfermedad, la fibrosis comienza a perder importancia y se comienza a producir la calcificación del tejido. En esta fase ocurre una diferenciación de las VICs a pre-osteoblastos, donde están implicadas otras vías de señalización.

La vía Notch es una de las vías de señalización más importante en esta enfermedad, ya que se ha demostrado en estudios anteriores que una haploinsuficiencia de Notch produce la calcificación de la válvula aórtica, y también se ha visto que la mayoría de los casos en los que se produce dicha calcificación, está asociada a mutaciones en algún elemento de esta vía [40]. Como se puede observar en la Figura 2, la vía Notch interviene en la expresión de los factores de transcripción Her y Hey, que son los responsables de inhibir la expresión de RUNX-2, y actúa bloqueando la entrada de $\beta$-catenina en el núcleo, proteína que es capaz de activar a RUNX-2.

Otra vía muy importante en esta patología es la vía de BMP2, que a través de la vía canónica interacciona con las SMAD 1/5/8 y estas se encargan de activar la transcripción de RUNX2. Se ha demostrado que en los casos en los que se produce la calcificación de la válvula, esta vía se encuentra híper-activada, lo que conlleva a que el factor RUNX2 se encuentre sobre-expresado dando lugar a la diferenciación de las VICs [40].

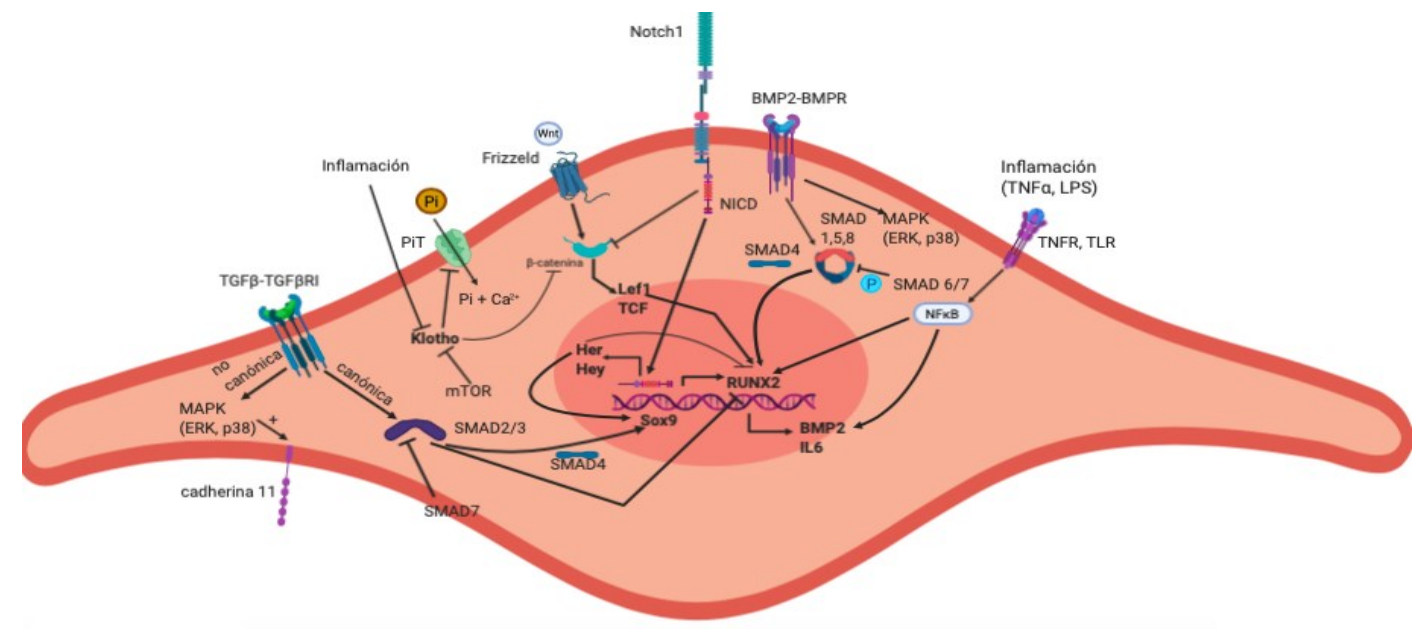

Figura 2. Vías de señalización que intervienen en el proceso de diferenciación a osteoblastos en las VICs

En los pre-osteoblastos, la presencia de BMP2 en el medio hace que se expresen numerosas proteínas de la matriz extracelular del tejido óseo, como la osteocalcina, la osteonectina, la osteopontina y también la fosfatasa alcalina, cuya función es eliminar grupos fosfatos de varios tipos de moléculas como nucleótidos y proteínas entre otras. Este fosfato inorgánico que se genera, podría reaccionar con los iones calcio y formar sales que precipitarán y producirán la calcificación [44].

Para evitar el fenotipo pro-calcificatorio de las células de la válvula, se identificaron dos posibles dianas terapéuticas que, como ya se ha nombrado anteriormente, están implicadas en la diferenciación de las células VICs a osteoblastos. Las dianas escogidas fueron el receptor de BMP2 (BMPR), que da lugar a la activación de una de las vías más expresadas en la EVAC, y el factor de transcripción RUNX-2, que como ya se ha comentado es el factor de transcripción más implicado en el desarrollo de la enfermedad.

Una vez determinadas las dianas terapéuticas, proponemos que el mejor mecanismo de acción para atacarlas sería emplear una terapia combinada en la que probaríamos un análogo sintético que actúe como agonista inverso de BMP2 y un miRNA contra RUNX-2. De esta forma se inhibirá el receptor BMPR y, por tanto, se bloqueará la vía de BMP2. Al mismo tiempo, el miRNA contra el RNAm de RUNX-2 bloqueará de esta forma su expresión. La elección de la terapia combinada se debe a que, como se ve en la Figura 2, no solo la vía de BMP2 estimula la síntesis de este factor de transcripción, por lo que un único tratamiento contra BMPR no sería suficiente para frenar la calcificación. 
Para la internalización de ambos fármacos de forma específica en las células de interés, proponemos como vehículos de direccionamiento liposomas que poseen aptámeros anclados a su superficie. Se ha visto en trabajos anteriores, que los liposomas se introducen en el interior de las células por endocitosis, estos también nos sirven para proteger a los fármacos y que de esta forma puedan llegar a su lugar de acción sin sufrir ninguna modificación [45]. Los aptaméros son ácidos nucleicos de cadena sencilla, con una estructura tridimensional que les permite unirse con alta afinidad y especificidad a las moléculas diana [46].

\section{Diseño del fármaco y ensayos biológicos}

\section{Diseño de análogos sintéticos de BMP2}

Para el diseño de análogos sintéticos de BMP2 utilizamos el programa de modelado molecular "pymol" [47] donde observamos las interacciones entre el receptor BMPR y el ligando BMP2.

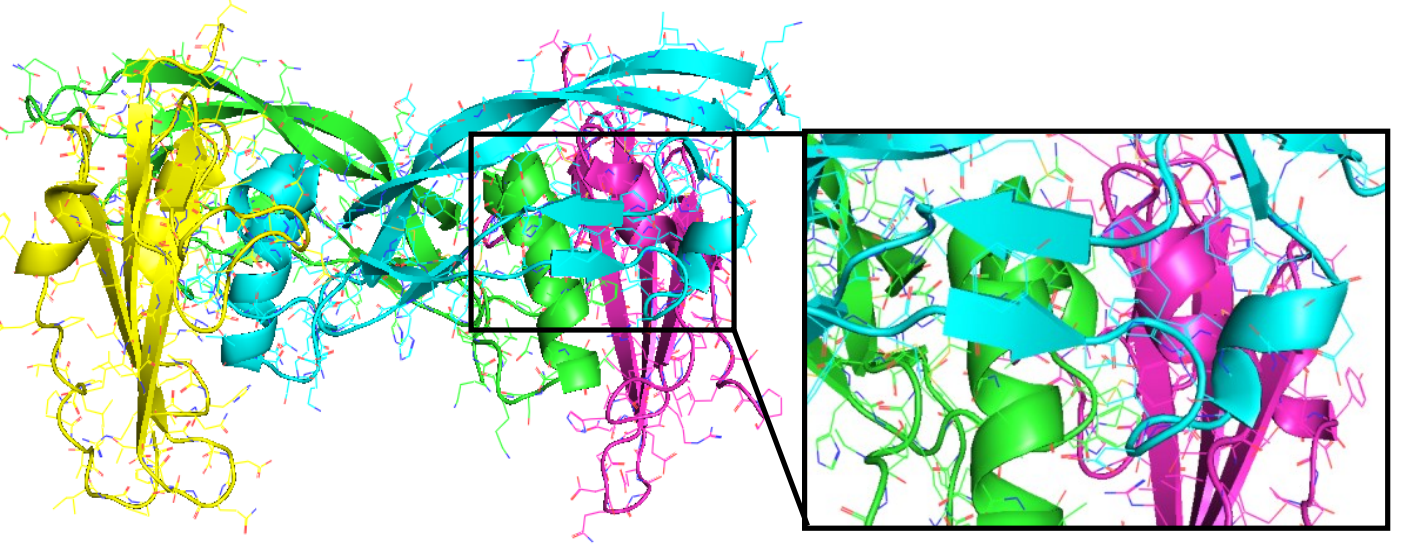

Figura 3. Ampliación de los posibles sitios de interacción entre el receptor, formado por las cadenas polipeptídicas amarilla y rosa, y el ligando BMP2, formado por las cadenas verde y azul.

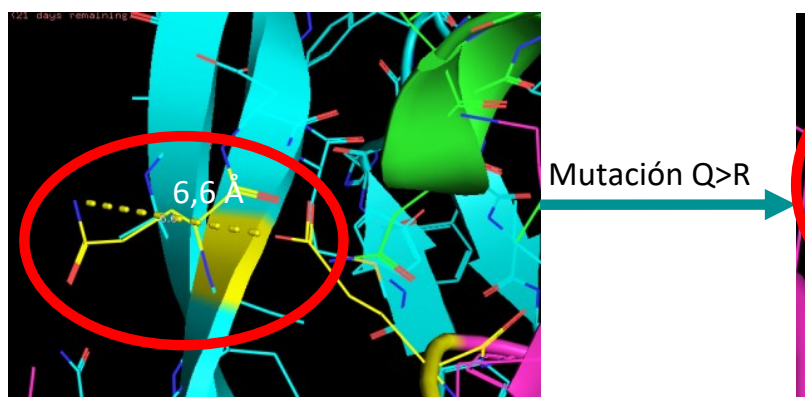

A

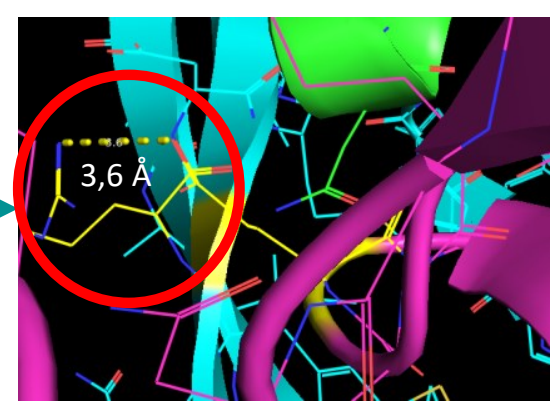

B

Figura 4. Ejemplo 1 de mutagénesis dirigida. A) Interacción entre el glutámico 81 del receptor y la glutamina 104 del ligando formando un posible puente de hidrógeno. B) Mutación de la glutamina por una arginina que posee una carga positiva que podría interaccionar electrostáticamente con el gutámico creando una interacción más fuerte. Se puede observar una disminución de la distancia de la interacción entre los aminoácidos.
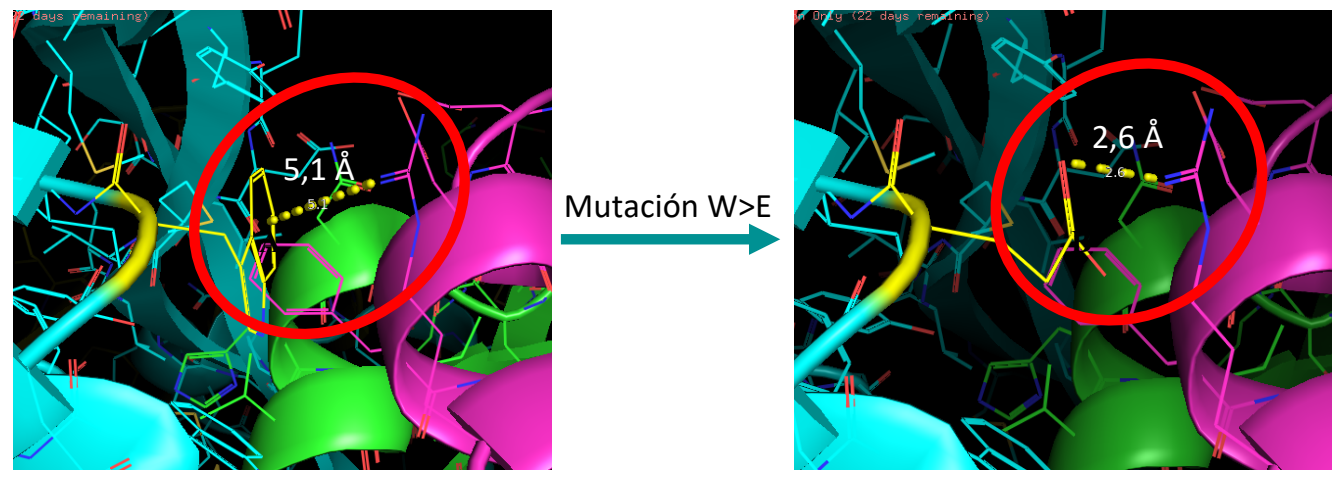

Figura 5. Ejemplo 2 de mutagénesis dirigida. A) Distancia entre el triptófano 31 del ligando y la arginina 88 del receptor. B) Mutación del W por un glutámico para crear de una nueva interacción electrostática observando una disminución de la distancia entre los aminoácidos. 
Estudiamos de forma racional los sitios de unión entre el BMPR y BMP2, como es la zona recuadrada de la Figura 3, y realizamos una mutagénesis dirigida de aminoácidos para mejorar las interacciones entre ligando y receptor. Para ello se realiza un estudio racional "in sílico" en el que se tendrán en cuenta las siguientes propiedades moleculares: una distancia menor entre los aminoácidos del receptor y ligando, que podría deberse a una mayor intensidad en las interacciones de hidrógeno o electrostáticas entre aminoácidos polares, e interacciones pi entre aminoácidos con anillos aromáticos [48]; además de tener en cuenta la formación del rotámero más estable que no interfiera en la estructura de la proteína.

De esta manera, modificando más aminoácidos y combinando las distintas mutaciones obtendríamos una biblioteca de análogos sintéticos de BMP2 para probar en nuestros ensayos de cribado con el objetivo de buscar péptidos miméticos de BMP2 que tengan una mayor afinidad por el receptor que el ligando fisiológico y que, a su vez, inhiban esta vía de señalización.

\section{Creación de una línea celular}

Se diseña una línea celular con el objetivo de detectar aquellos análogos sintéticos de BMP2 que inhiban al receptor. Para ello, las VICs son transfectadas con tres plásmidos. El primero de ellos, un plásmido multicopia, presenta el gen que expresa el receptor de BMP2 para conseguir una sobreexpresión del mismo y asegurarse de que los cambios detectados sean dependientes de esa ruta. El segundo contendrá un promotor inducible por RUNX-2 seguido de un gen que codifica para un miRNA de la luciferasa. Y un tercer plásmido codificará para la luciferasa de forma constitutiva, utilizando para ello un promotor inducible constitutivamente activo. Esta línea celular permitirá que, en presencia de BMP2, dicha ruta se vea sobre-expresada, lo que llevará a un incremento de RUNX-2, a la expresión del miRNA de la luciferasa y, como consecuencia, a la ausencia de luz. Sin embargo, si se consigue inhibir la ruta de BMP2 con alguno de los análogos sintéticos diseñados, no habrá expresión de RUNX-2, se sintetizará la luciferasa y, por tanto, se detectará luz (Figura 6).

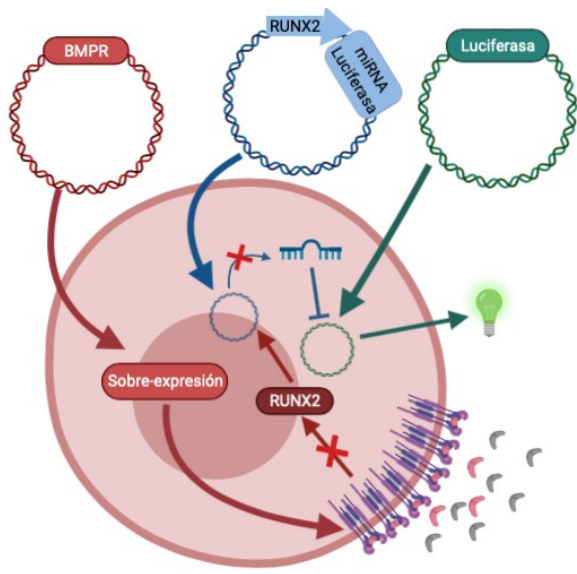

Figura 6. Detección de la inhibición de la ruta de BMP2.

La presencia de un análogo sintético de BMP2 capaz de inhibir la ruta, llevará a la reducción de los niveles del miRNA contra la luciferasa al no expresarse RUNX-2. Por lo tanto, se sintetizará la luciferasa y se podrá detectar luz.

\section{Ensayo de actividad biológica:}

La línea celular diseñada es tratada con concentraciones constantes de BMP2 fisiológico y junto con los distintos péptidos miméticos de BMP2. De tal forma que, si alguno de esos análogos sintéticos de BMP2 es capaz de inhibir al receptor, incluso en presencia del ligando fisiológico, entonces se detectará luz. Esta emisión de luz es directamente proporcional a la inhibición de la ruta provocada por el análogo sintético de BMP2, obteniendo así los mejores "hits". Una vez sintetizados los péptidos "hit" de nuestro proyecto, volveríamos a realizar modificaciones sobre ellos y a realizar de nuevo este estudio para ver si podemos mejorar su actividad realizando así el paso "hit to lead".

\section{Ensayo de afinidad}

Una vez seleccionados aquellos péptidos miméticos que presentan una mayor actividad inhibitoria, se dispone a realizar un ensayo para determinar aquellos que tienen mayor afinidad por el receptor. Para ello se fijará en una placa multipocillo el receptor de BMP2 aislado. Sobre dichos pocillos se añade BMP2 fisiológico fusionado a GFP (Green Fluorescent Protein) [49] a una concentración constante junto con el análogo sintético en concentraciones crecientes, dejando una columna de la placa multipocillos como control del experimento, añadiendo únicamente BMP2 "wild type" unido a GFP para ver el máximo de intensidad de fluorescencia. Una vez añadidos los análogos e incubados será necesario lavar con PBS (Phosphate Buffer Saline) para eliminar aquellos ligandos que queden sin unir y se medirá la 
fluorescencia. Cuanto menor sea la afinidad del péptido mimético sobre el receptor mayor será la fluorescencia observada. En definitiva, se seleccionarán aquellos análogos sintéticos que presentan una menor fluorescencia con una menor concentración utilizada, obteniendo así los candidatos de nuestro proyecto que pasarían al ensayo terapéutico. Para la selección de los candidatos, elegiremos aquellos que nos proporcionen un menor valor de $\mathrm{IC}_{50}$, la $\mathrm{IC}_{50}$ nos proporciona la concentración a la cual la vía se encuentra inhibida un $50 \%$, cuanta menor $\mathrm{IC}_{50}$, menor concentración se necesitará del análogo sintético para conseguir una mayor inhibición.

\section{Ensayo de internalización de los liposomas}

Para la administración del principio activo se utilizan los liposomas como vehículos de direccionamiento hacia la diana terapéutica. Como hemos mencionado anteriormente, los liposomas se introducen en el interior de la célula por endocitosis y se caracterizan por ser vesículas sintéticas formadas por una bicapa lipídica que crea un ambiente hidrofílico en su interior. Pueden ser utilizados en biomedicina como vehículos de direccionamiento y van a permitir el transporte de nuestros fármacos polares en su interior. Para comprobar la internalización de los liposomas terapéuticos se diseñan liposomas en cuya superficie se exponen aptámeros, que reconocen los biomarcadores propios de las VICs transformadas o en proceso de diferenciación (mencionados anteriormente), y uno de los análogos sintéticos de BMP2 seleccionado previamente (candidatos) (Figura 7). En su interior se introduce una molécula orgánica fluorescente (CY3) [50]. Si los liposomas terapéuticos son fagocitados se detectará fluorescencia en el interior de las células.

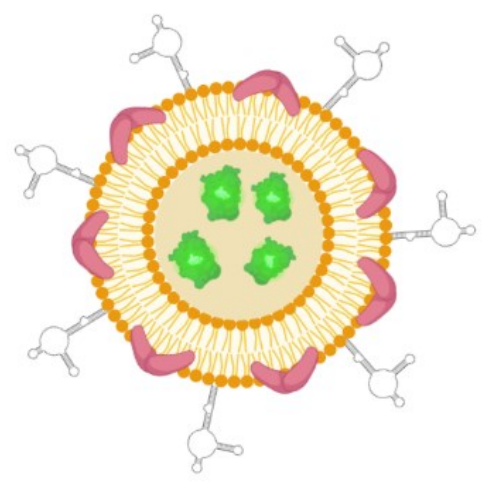

Figura 7. Diseño de los liposomas para el estudio de su internalización en las células. Expresan aptámeros (gris) y un análogo sintético de BMP2 (rosa) en su superficie. En su interior se introduce la proteína fluorescente CY3 (verde).

Para este ensayo utilizaremos distintas líneas celulares: VICs, células endoteliales de la válvula (similares a las VICs) y células pancreáticas (distintas a las VICs). Se hará un ensayo con cada una de estas líneas por separado y otro con todas ellas juntas para comprobar la selectividad de los liposomas hacia nuestras células diana (las VICs).

Esta selectividad va a venir dada tanto por el análogo sintético de BMP2 utilizado, puesto que posee una alta afinidad por BMPR que estará sobreexpresado en las VICs, como por los aptámeros diseñados. Los aptámeros son cadenas monocatenarias de ácidos nucleicos con estructura tridimensional que les permite unirse con una alta afinidad a moléculas diana específicas [46]. Algunas de las moléculas diana que se podrían seleccionar para que los aptámeros se unan específicamente son: el receptor RANK, asmootelina, el receptor Notch1 mutado o BMPR. Ya que estas moléculas se pueden encontrar sobreexpresadas en la EVAC, incluso en un momento previo al diagnóstico de la enfermedad cuando las células VICs empiezan a adquirir un fenotipo de mio-fibroblasto pro-calcificatorio.

Para la introducción de los liposomas en el organismo se utilizaría la vía intravenosa con un dispositivo similar al de la insulina, de esta manera se pretende mejorar su direccionamiento hacia el tejido de interés y su biodisponibilidad.

\section{Ensayo terapéutico:}

En este ensayo se analiza el efecto de los péptidos miméticos (candidatos) y un miRNA-138 específico de RUNX-2, de forma individual y en sinergia. En estudios previos se ha demostrado que el miRNA-138 disminuye la calcificación de las VICs [51], por lo que se seleccionó como inhibidor de RUNX-2.

Para poder analizar los efectos inhibitorios de dichas moléculas sobre la ruta de BMP2 y el proceso de calcificación se realizan los siguientes subensayos:

i. Condiciones basales, VICs que sobreexpresan BMPR incubadas con BMP2 "wild type" en concentraciones fisiológicas

ii. Condiciones basales tratadas con liposomas que exponen el péptido mimético 
iii. Condiciones basales tratadas con liposomas que contienen el miRNA-138

iv. Condiciones basales tratadas con liposomas que poseen tanto el péptido mimético como el miRNA138.

Tras 2 y 24 horas de tratamiento se analizará la cantidad de RUNX-2, receptor de la hormona paratiroidea (PTHR) y osteopontina mediante el uso de "quantum dots" (QD). Los QD son esferas que emiten fluorescencia de forma muy intensa y estable convirtiéndolos en una técnica de multiplexación altamente sensible [52]. Estos QD, además de emitir en longitudes de onda diferentes, están unidos a anticuerpos primarios que detectan de forma selectiva los biomarcadores mencionados anteriormente. Concretamente RUNX-2 se utilizará como marcador de la activación de la vía de BMP2 siendo detectado en rojo (620nm). PTHR y osteopontina se emplearán como marcadores de diferenciación de las VICs hacia un fenotipo osteogénico, detectándose en verde $(520 \mathrm{~nm})$ y en azul $(488 \mathrm{~nm})$ respectivamente. La energía de emisión es cuantificada mediante microscopía de fluorescencia, pudiendo determinar la cantidad de biomarcadores presentes en las células.

Los resultados que cabría esperar de los ensayos ii y iii es una reducción parcial de la señal emitida por los QD, debido a que han sido expuestos a un único tratamiento. Mientras que en el ensayo iv se esperaría la potenciación de la acción combinada de ambos fármacos, y, por lo tanto, una reducción aún mayor e incluso total de la emisión.

\section{Conclusiones y perspectivas de futuro}

Con este estudio lo que se pretende es el diseño de una terapia alternativa, al actual reemplazamiento valvular quirúrgico, que elimine todos los riesgos asociados a una cirugía como el rechazo, el postoperatorio, una infección, etc. Este tratamiento mínimamente invasivo evitaría el remodelado valvular asegurando la funcionalidad de la válvula aórtica a largo plazo de forma fisiológica. Además, sería una opción para aquellas personas que no puedan someterse a una operación.

En cuanto a las perspectivas de futuro de esta terapia, se desearía diseñar un inyector de tratamiento fácil de usar, portátil y compacto que permita la autonomía del paciente. Incluso se podría llegar a plantear una administración oral del fármaco mejorando todavía más la calidad de vida del paciente para lo que se requiere de una mayor investigación de la absorción de los liposomas a nivel intestinal. De forma complementaria a nuestra terapia combinada, es necesaria la búsqueda de biomarcadores en sangre que permitan una detección de la enfermedad a tiempo, antes de que la calcificación de la válvula ya sea irreversible.

\section{Referencias}

1. Coffey S, Cairns BJ, Iung B. The modern epidemiology of heart valve disease. Heart. 2016;102(1):75-85.

2. Mathieu P, Arsenault BJ. CAVD: Civilization aortic valve disease. Eur Heart J. 2017;38(28):2198-200.

3. Vyas P, Hutcheson JD, Aikawa E. Calcific Aortic Valve Disease: Pathobiology, Basic Mechanisms, and Clinical Strategies. Adv Hear Valve Biomech. 2018;153-79.

4. Stella JA, Sacks MS. On the biaxial mechanical properties of the layers of the aortic valve leaflet. J Biomech Eng. 2007;129(5):757-66.

5. Vesely I, Noseworthy R. Micromechanics of the fibrosa and the ventricularis in aortic valve leaflets. J Biomech. 1992;25(1).

6. Merryman WD, Shadow Huang HY, Schoen FJ, Sacks MS. The effects of cellular contraction on aortic valve leaflet flexural stiffness. J Biomech. 2006;39(1):88-96.

7. Zhao R, Sider KL, Simmons CA. Measurement of layer-specific mechanical properties in multilayered biomaterials by micropipette aspiration. Acta Biomater [Internet]. 2011;7(3):12207.

8. Schoen FJ. Evolving concepts of cardiac valve dynamics: The continuum of development, functional structure, pathobiology, and tissue engineering. Circulation. 2008;118(18):1864-80.

9. Sacks MS, Smith DB, Hiester ED. The aortic valve microstructure: Effects of transvalvular pressure. J Biomed Mater Res. 1998;41(1):131-41.

10. Stella JA, Liao J, Sacks MS. Time-dependent biaxial mechanical behavior of the aortic heart valve leaflet. J Biomech. 2007;40(14):3169-77.

11. Cimini M, Rogers KA, Boughner DR. Smoothelin-positive cells in human and porcine semilunar valves. Histochem Cell Biol. 2003;120(4):307-17. 
12. Chester AH, Kershaw JDB, Sarathchandra P, Yacoub MH. Localisation and function of nerves in the aortic root. J Mol Cell Cardiol. 2008;44(6):1045-52.

13. Liu AC, Joag VR, Gotlieb AI. The emerging role of valve interstitial cell phenotypes in regulating heart valve pathobiology. Am J Pathol. 2007;171(5):1407-18.

14. Chen JH, Yip CYY, Sone ED, Simmons CA. Identification and characterization of aortic valve mesenchymal progenitor cells with robust osteogenic calcification potential. Am J Pathol. 2009;174(3):1109-19.

15. Durbin AD, Gotlieb AI. Advances towards understanding heart valve response to injury. Cardiovasc Pathol. 2002;11(2):69-77.

16. Merryman WD, Lukoff HD, Long RA, Engelmayr GC, Hopkins RA, Sacks MS. Synergistic effects of cyclic tension and transforming growth factor- $\beta 1$ on the aortic valve myofibroblast. Cardiovasc Pathol. 2007;16(5):268-76.

17. Eloy JO, Petrilli R, Trevizan LNF, Chorilli M. Immunoliposomes: A review on functionalization strategies and targets for drug delivery. Colloids Surfaces B Biointerfaces. 2017;159:454-67.

18. Lopez-Berestein G, Fainstein V, Hopfer R, Mehta K, Sullivan MP, Keating M, et al. Liposomal amphotericin $\mathrm{B}$ for the treatment of systemic fungal infections in patients with cancer: A preliminary study. J Infect Dis. 1985;151(4):704-10.

19. Lee WH, Loo CY, Traini D, Young PM. Nano- and micro-based inhaled drug delivery systems for targeting alveolar macrophages. Expert Opin Drug Deliv. 2015;12(6):1009-26.

20. Ternullo S, de Weerd L, Holsæter AM, Flaten GE, Škalko-Basnet N. Going skin deep: A direct comparison of penetration potential of lipid-based nanovesicles on the isolated perfused human skin flap model. Eur J Pharm Biopharm. 2017;121(June):14-23.

21. Wu W, Lu Y, Qi J. Oral delivery of liposomes. Ther Deliv. 2015;6(11):1239-41.

22. Uhl P, Pantze S, Storck P, Parmentier J, Witzigmann D, Hofhaus G, et al. Oral delivery of vancomycin by tetraether lipid liposomes. Eur J Pharm Sci. 2017;108:111-8.

23. Aikawa E, Nahrendorf M, Sosnovik D, Lok VM, Jaffer FA, Aikawa M, et al. Multimodality molecular imaging identifies proteolytic and osteogenic activities in early aortic valve disease. Circulation. 2007;115(3):377-86.

24. Aikawa E, Otto CM. Look more closely at the valve: Imaging calcific aortic valve disease. Circulation. 2012;125(1):9-11.

25. New SEP, Aikawa E. Molecular imaging insights into early inflammatory stages of arterial and aortic valve calcification. Circ Res. 2011;108(11):1381-91.

26. Helske S, Kupari M, Lindstedt KA, Kovanen PT. Aortic valve stenosis: An active atheroinflammatory process. Curr Opin Lipidol. 2007;18(5):483-91.

27. Aronow WS, Schwartz KS, Koenigsberg M. Correlation of serum lipids, calcium, and phosphorus, diabetes mellitus and history of systemic hypertension with presence or absence of calcified or thickened aortic cusps or root in elderly patients. Am J Cardiol. 1987;59(9):998-9.

28. Gerber Y, Goldbourt U, Feinberg MS, Segev S, Harats D. Are triglyceride-rich lipoproteins associated with aortic valve sclerosis? A preliminary report. Atherosclerosis. 2003;170(2):301-5.

29. Adeney KL, Siscovick DS, Ix JH, Seliger SL, Shlipak MG, Jenny NS, et al. Association of serum phosphate with vascular and valvular calcification in moderate CKD. J Am Soc Nephrol. 2009;20(2):381-7.

30. Linefsky JP, OBrien KD, Katz R, De Boer IH, Barasch E, Jenny NS, et al. Association of serum phosphate levels with aortic valve sclerosis and annular calcification. J Am Coll Cardiol. 2011;58(3):291-7.

31. Linefsky JP, O’Brien KD, Sachs M, Katz R, Eng J, Michos ED, et al. Serum phosphate is associated with aortic valve calcification in the Multi-ethnic Study of Atherosclerosis (MESA). Atherosclerosis. 2014;233(2):331-7.

32. Civitelli R, Armamento-Villareal R, Napoli N. Bone turnover markers: Understanding their value in clinical trials and clinical practice. Osteoporos Int. 2009;20(6):843-51.

33. Schäfer C, Heiss A, Schwarz A, Westenfeld R, Ketteler M, Floege J, et al. The serum protein $\alpha 2$ - Heremans-Schmid glycoprotein fetuin-A is a systemically acting inhibitor of ectopic calcificati.pdf. J Clin Invest. 2003;112(3):357-66. 
34. Wang AYM, Woo J, Lam CWK, Wang M, Chan IHS, Gao P, et al. Associations of serum fetuinA with malnutrition, inflammation, atherosclerosis and valvular calcification syndrome and outcome in peritoneal dialysis patients. Nephrol Dial Transplant. 2005;20(8):1676-85.

35. Ix JH, Chertow GM, Shlipak MG, Brandenburg VM, Ketteler M, Whooley MA. Association of fetuin-A with mitral annular calcification and aortic stenosis among persons with coronary heart disease: Data from the heart and soul study. Circulation. 2007;115(19):2533-9.

36. Libby P, Ridker PM. Inflammation and atherosclerosis: Role of C-reactive protein in risk assessment. Am J Med. 2004;116(6 SUPPL. 1):9-16.

37. Freeman R V., Otto CM. Spectrum of calcific aortic valve disease: Pathogenesis, disease progression, and treatment strategies. Circulation. 2005;111(24):3316-26.

38. Towler DA. Molecular and cellular aspects of calcific aortic valve disease. Circ Res. 2013;113(2):198-208.

39. Khera A, De Lemos JA, Peshock RM, Lo HS, Stanek HG, Murphy SA, et al. Relationship between C-reactive protein and subclinical atherosclerosis: The Dallas Heart Study. Circulation. 2006;113(1):38-43.

40. Rutkovskiy A, Malashicheva A, Sullivan G, Bogdanova M, Kostareva A, Stensløkken KO, et al. Valve interstitial cells: The key to understanding the pathophysiology of heart valve calcification. J Am Heart Assoc. 2017;6(9):1-23.

41. Lerman DA, Prasad S, Alotti N. Calcific aortic valve disease: Molecular mechanisms and therapeutic approaches. Eur Cardiol Rev . 2015;10(2):108-12.

42. Osta B, Benedetti G, Miossec P. Classical and paradoxical effects of TNF- $\alpha$ on bone homeostasis. Front Immunol. 2014;5(FEB):1-9.

43. Voelkl J, Lang F, Eckardt KU, Amann K, Kuro-o M, Pasch A, et al. Signaling pathways involved in vascular smooth muscle cell calcification during hyperphosphatemia. Cell Mol Life Sci. 2019;76(11):2077-91.

44. Rutkovskiy A, Stensløkken K-O, Vaage IJ. Osteoblast Differentiation at a Glance. Med Sci Monit Basic Res. 2016;22:95-106.

45. Nisini R, Poerio N, Mariotti S, De Santis F, Fraziano M. The multirole of liposomes in therapy and prevention of infectious diseases. Front Immunol. 2018;9(FEB):1-23.

46. Moosavian SA, Sahebkar A. Aptamer-functionalized liposomes for targeted cancer therapy. Cancer Lett. 2019;448(January):144-54.

47. Lill MA, Danielson ML. Computer-aided drug design platform using PyMOL. J Comput Aided Mol Des. 2011;25(1):13-9.

48. Biedermann F, Schneider HJ. Experimental Binding Energies in Supramolecular Complexes. Chem Rev. 2016;116(9):5216-300.

49. Gerdes HH, Kaether C. Green fluorescent protein: Applications in cell biology. FEBS Lett. 1996;389(1):44-7.

50. Zhang W, Peng F, Zhou T, Huang Y, Zhang L, Ye P, et al. Targeted delivery of chemically modified anti-miR-221 to hepatocellular carcinoma with negatively charged liposomes. Int $\mathrm{J}$ Nanomedicine. 2015;10:4825-36.

51. Lu P, Yin B, Liu L. MicroRNA-138 suppresses osteoblastic differentiation of valvular interstitial cells in degenerative calcific aortic valve disease. Int Heart J. 2019;60(1):136-44.

52. Jamieson T, Bakhshi R, Petrova D, Pocock R, Imani M, Seifalian AM. Biological applications of quantum dots. Biomaterials. 2007;28(31):4717-32. 